\title{
Synthesis and biological evaluation of small molecule modulators of CDK8/Cyclin C complex with phenylaminoquinoline scaffold
}

\author{
Mohammad M. Al-Sanea ${ }^{\text {Corresp. } 1}$ \\ ${ }^{1}$ Pharmaceutical Chemistry Department, College of Pharmacy, Jouf University, Sakaka, Aljouf, Saudi Arabia \\ Corresponding Author: Mohammad M. Al-Sanea \\ Email address: mmalsanea@ju.edu.sa
}

Background. $\mathrm{CDK} 8 / \mathrm{CycC}$ complex has kinase activity towards the carboxyterminal domain of RNA polymerase II, and contributes to the regulation of transcription via association with the mediator complex. Different human malignancies, mainly colorectal and gastric cancers, were produced as a result of overexpression of CDK8/CycC in the mediator complex. Therefore, CDK8/CycC complex represents as a cancer oncogene and it has become a potential target for developing CDK8/CycC modulators.

Methods. A series of nine 4-phenylaminoquinoline scaffold-based compounds 5a-i were synthesized, and biological evaluation of this series was performed as potential inhibitors of CDK8/CycC complex.

Results. The scaffold substituent effects on the intrinsic inhibitory activity toward CDK8/CycC complex are addressed trying to present a novel outlook of CDK8/CycC Complex inhibitors with 4-

phenylaminoquinoline scaffold in cancer therapy. The secondary benzenesulfonamide analogues proved to be the most potent compounds in suppressing CDK8/CycC enzyme, whereas, their primary benzenesulfonamide analogues showed inferior activity. Moreover, the benzene reversed sulfonamide analogues were totally inactive.

Discussion. The titled scaffold showed promising inhibitory activity data and there is a crucial role of un/substituted sulfonamido group for CDK8/CycC complex inhibitory activity. Compound $\mathbf{5 d}$ showed submicromolar potency against CDK8/CycC $\left(\mathrm{IC}_{50}=0.639 \mu \mathrm{M}\right)$ and it can be used for further investigations and to design another larger library of phenylaminoquinoline scaffold-based analogues in order to establish detailed SARs. 


\section{Synthesis and Biological Evaluation of small molecule}

3 modulators of CDK8/Cyclin C Complex with

4 Phenylaminoquinoline Scaffold

5

6

7

8

9

Mohammad Mahmoud Al-Sanea

Pharmaceutical Chemistry Department, College of Pharmacy, Jouf University, Sakaka, Aljouf 2014, Saudi Arabia

Corresponding Author:

Mohammad Al-Sanea

Pharmaceutical Chemistry Department, College of Pharmacy, Jouf University, Sakaka, Aljouf 2014, Saudi Arabia

Email address: mmalsanea@ju.edu.sa

\section{Abstract}

Background. $\mathrm{CDK} 8 / \mathrm{CycC}$ complex has kinase activity towards the carboxyterminal domain of RNA polymerase II and contributes to the regulation of transcription via association with the mediator complex. Different human malignancies, mainly colorectal and gastric cancers, were produced as a result of overexpression of $\mathrm{CDK} 8 / \mathrm{CycC}$ in the mediator complex. Therefore, $\mathrm{CDK} 8 / \mathrm{CycC}$ complex represents a cancer oncogene and it has become a potential target for developing $\mathrm{CDK} 8 / \mathrm{CycC}$ modulators.

Methods. A series of nine 4-phenylaminoquinoline scaffold-based compounds 5a-i were synthesized, and the biological evaluation of this series was performed as potential inhibitors of CDK8/CycC complex.

Results. The scaffold substituent effects on the intrinsic inhibitory activity toward CDK8/CycC complex are addressed trying to present a novel outlook of $\mathrm{CDK} 8 / \mathrm{CycC}$ Complex inhibitors with 4-phenylaminoquinoline scaffold in cancer therapy.

The secondary benzenesulfonamide analogues proved to be the most potent compounds in suppressing $\mathrm{CDK} 8 / \mathrm{CycC}$ enzyme, whereas, their primary benzenesulfonamide analogues showed inferior activity. Moreover, the benzene reversed sulfonamide analogues were inactive. Discussion. The titled scaffold showed promising inhibitory activity data and there is a crucial role of un/substituted sulfonamido group for $\mathrm{CDK} 8 / \mathrm{CycC}$ complex inhibitory activity. Compound 5d showed submicromolar potency against $\mathrm{CDK} 8 / \mathrm{CycC}\left(\mathrm{IC}_{50}=0.639 \mu \mathrm{M}\right)$ and it can be used for further investigations and to design another larger library of phenylaminoquinoline scaffold-based analogues to establish detailed SARs. 
40

41

42

43

44

45

46

47

48

49

50

51

52

53

54

55

56

57

58

59

60

61

62

63

64

65

66

67

68

69

70

71

72

73

74

75

76

77

\section{Introduction}

Cyclin-dependent kinases (CDKs) drive cell cycle through phosphorylation of a variety of vital substrates (Satyanarayana \& Kaldis 2009). Association of CDKs with regulatory partners (cyclins) regulates CDKs activity (Obaya \& Sedivy 2002). Therefore, several cyclin/kinase complexes have been considered as essential for controlled cell proliferation (Sears \& Nevins 2002). Cyclin $\mathrm{C}$ is known to form a stable complex with $\mathrm{CDK} 8$ (CDK8/CycC complex). The kinase active complex is associated with direct phosphorylation activity towards gene-specific transcription factors, thus controls their downstream function (Nemet et al. 2014). Hence, $\mathrm{CDK} 8 / \mathrm{CycC}$ can modulate transcriptional output from distinct transcription factors involved in oncogenic control (Malik \& Roeder 2005). Recent evidence supports the idea that mediator complex-associated $\mathrm{CDK} 8 / \mathrm{CycC}$ has been involved in the regulation of multiple transcription pathways and implicated as an oncogene in colorectal and gastric cancers through activation of WNT signaling (Kim et al. 2006; Rzymski et al. 2015). CDK8 is amplified and overexpressed in colon, gastric, breast cancers and melanoma (Li et al. 2014a; Roninson et al. 2019). Accordingly, CDK8/CycC complex may represent a potential drug target for different kinds of human malignancies with reduced toxic effects on normal cells (Chen et al. 2019; He et al. 2019; Rzymski et al. 2015; Sánchez-Martínez et al. 2019; Schneider et al. 2013). Moreover, $\mathrm{CDK} 8 / \mathrm{CycC}$ complex plays several roles in modulating gene expression levels (Firestein et al. 2008; Knuesel et al. 2009a; Knuesel et al. 2009b; Li et al. 2014b).

Even though CDK inhibitors have been abundantly described, attempts of discovering selective CDK8 inhibitors have emerged as a promising strategy for cancer therapy as Pan-CDK inhibitor has shown narrow therapeutic window and potential risks (Al-Sanea et al. 2015; Al-Sanea et al. 2016b; Al-Sanea et al. 2015; Firestein et al. 2008; Kapoor et al. 2010; Xu \& Ji 2011). Such selective inhibitors allow cancer therapy by reducing mitogenic signals in several cancer cells (Adler et al. 2012; McDermott et al. 2017; Rzymski et al. 2015).

Several chemical scaffold-based small molecules have been applied for the design of CDK8 inhibitors. Among these scaffolds, quinoline and its bioisosteres have successively shown potent modulation of CDK8 activity. The steroidal natural product cortistatin A, which has quinoline moiety as a hinge component and steroidal core responsible for extensive intermolecular forces with the ATP-binding cavity, showed a highly potent ATP-competitive CDK8 inhibitory activity $\left(\mathrm{IC}_{50}=15 \mathrm{nM}\right)$ that exhibited anticancer activity in animal models of acute myeloid leukemia (AML) (Cee et al. 2009; Crown 2017; Pelish et al. 2015; Rzymski et al. 2017). Senexin B with 4aminoquinazoline scaffold showed potent CDK8 modulation with an $\mathrm{IC}_{50}$ value of $24 \mathrm{nM}$ (McDermott et al. 2017; Rzymski et al. 2015). In 2016, Schiemann et al. described new potent and selective $\mathrm{CDK} 8$ ligands with benzylindazole scaffold that showed an $\mathrm{IC}_{50}$ value against CDK8 of $10 \mathrm{nM}$ (Schiemann et al. 2016). Besides, many well-known kinase ligands such as sorafenib and imatinib represent another type of potent CDK8 inhibitors with different binding modes (Chen et al. 2019; Schneider et al. 2011). 
78 It is noteworthy that the kinase activity of CDK8 is affected by substrate binding and association

79 with other mediator complex members as well. By utilizing a scaffold hopping strategy on the

80 aforementioned quinoline isosteres, a series of new phenylaminoquinoline derivatives with

81 sulphonamide moiety at position 3 in terminal phenyl ring was designed, synthesized and

82 pharmacologically evaluated as potential small molecule modulators of $\mathrm{CDK} 8 / \mathrm{CycC}$ complex.

83

\section{Materials \& Methods}

85 All chemical reagents and solvents were of analytical grade, purchased from commercial

86

87

88

89

90

91

92

93

94

95

96

97

98

99

100

101

102

103

104

105

106

107

108

109

110

111

112

113

114

115

116

117 suppliers (Sigma-Aldrich and Alfa-aesar) and utilized to accomplish this work as received. All reactions were carried out in a dry nitrogen atmosphere. The microwave-assisted synthesis was carried out in a Biotage Initiator apparatus operating in single mode, the microwave cavity producing controlled irradiation at $2.45 \mathrm{GHz}$ (Biotage $\mathrm{AB}$, Uppsala, Sweden). The reactions were run in sealed vessels. These experimentations were carried out by employing magnetic stirring and a fixed hold time applying variable power to reach (during 1-2 min) and then keep the desired temperature in the vessel for the preset time. On the reactor vial glass, an IR sensor was applied to monitor the temperature. The NMR spectra were obtained on Bruker Avance 400 (400 MHz ${ }^{1} \mathrm{H}$ and $100.6 \mathrm{MHz}{ }^{13} \mathrm{C}$ NMR). Column chromatography was performed on Merck Silica Gel 60 (230-400 mesh). Thin layer chromatography (TLC) was performed using sheets pre-coated with silica gel 60 F254 supplied from Merck. The purity of compounds was determined by analytical high performance liquid chromatography (HPLC) using a Water ACQUITY UPLC (CORTECSTM) with C18 column $(2.1 \mathrm{~mm} \times 100 \mathrm{~mm} ; 1.6 \mu \mathrm{m})$ at $40{ }^{\circ} \mathrm{C}$. HPLC data were noted using parameters as follows: $0.1 \%$ formic acid in water and $0.1 \%$ formic acid in methanol and flow rate of $0.3 \mathrm{~mL} / \mathrm{min}$. Waters ACQUITY UPLC BEH C18 $1.7 \mu-\mathrm{Q}-$ TOF SYNAPT G2-Si High Definition Mass Spectrometry was used to obtain high-resolution spectra. Compounds 3-4 and 5a-c, i were previously reported (Al-Sanea et al. 2019).

Common procedures for the synthesis of key intermediates $\mathbf{4 a - d .}$

A solution of intermediated $\mathbf{3 a}-\mathbf{d}(1.0 \mathrm{mmol})$ in $\mathrm{POCl}_{3}(6 \mathrm{~mL})$ was refluxed for $1 \mathrm{~h}$. Evaporation of the mixture was performed in vacuo and the residue was extracted with methylene chloride, aqueous ammonia, and crushed ice. The methylene chloride layer was dried over anhydrous $\mathrm{Na}_{2} \mathrm{SO}_{4}$ and concentrated. Column chromatography $\left(\mathrm{SiO}_{2}, \mathrm{EA}: n-\mathrm{Hex}\right)$ was applied to purify the residue to get compounds $\mathbf{4 a - c}$ (Scheme 1)

Common procedures for the synthesis of the target compounds $\mathbf{5 a - i}$

To a microwave vial, were sequentially added the appropriate intermediated $4 \mathbf{a - c}(0.21 \mathrm{mmol})$, 3-amino- $N$-methylbenzenesulfonamide ( $0.04 \mathrm{gm}, 0.21 \mathrm{mmol})$, or $\mathrm{N}$-(3phenyl)methanesulfonamide ( $0.04 \mathrm{gm}, 0.21 \mathrm{mmol}), 3$-aminobenzenesulfonamide $(0.036 \mathrm{gm}$, $0.21 \mathrm{mmol})$, and absolute ethyl alcohol $(12 \mathrm{~mL})$. The microwave vial was sealed and heated under microwave conditions at $150^{\circ} \mathrm{C}$ for $30 \mathrm{~min}$. The reaction mixture was evaporated in vacuo and the residue was extracted with ethyl acetate and $\mathrm{NaHCO}_{3}$ (aq). The ethyl acetate layer was 
118 dried over anhydrous $\mathrm{Na}_{2} \mathrm{SO}_{4}$ and concentrated. The residue was purified by column

119 chromatography $\left(\mathrm{SiO}_{2}, \mathrm{EA}: n\right.$-Hex) to provide quinolines 5a-i (Scheme 1).

120

121

Ethyl 6-Bromo-4-(3-sulfamoyl-phenylamino)-quinoline-3-carboxylate (5a)

122

Yellow solid, yield: 71\%, mp: $235.6-237.2{ }^{\circ} \mathrm{C} ;{ }^{1} \mathrm{H}$ NMR (DMSO-d6, $\left.400 \mathrm{MHz}\right) \delta p p m: 1.07$ (t,

123 $\left.3 \mathrm{H}, J=5.6 \mathrm{~Hz}, \mathrm{CH}_{2} \mathrm{CH}_{3}\right), 3.88\left(\mathrm{q}, 2 \mathrm{H}, J=5.6 \mathrm{~Hz}, \mathrm{CH}_{2} \mathrm{CH}_{3}\right), 7.17(\mathrm{~s}, 1 \mathrm{H}), 7.36(\mathrm{~s}, 2 \mathrm{H}$,

124 $\left.\mathrm{SO}_{2} \mathrm{NH}_{2}\right), 7.45-7.51(\mathrm{~m}, 3 \mathrm{H}), 7.94(\mathrm{~s}, 2 \mathrm{H}), 8.53(\mathrm{~s}, 1 \mathrm{H}), 8.92(\mathrm{~s}, 1 \mathrm{H}), 9.71(\mathrm{~s}, 1 \mathrm{H}, \mathrm{NH}) ;{ }^{13} \mathrm{C}$ NMR

125 (DMSO-d6, $100 \mathrm{MHz}$ ) $\delta p p m: 14.22\left(\mathrm{CH}_{3}\right), 61.49\left(\mathrm{CH}_{2}\right), 111.61$ (phenyl C-2), 116.40 (phenyl C-4), 119.81 (phenyl C-6), 119.95 (quinoline C-6), 121.47 (quinoline C-3), 123.08 (quinoline C10), 126.60 (quinoline $\mathrm{C}-5$ ), 130.21 (phenyl C-5), 132.13 (quinoline $\mathrm{C}-8$ ), 134.76 (quinoline $\mathrm{C}$ -

128

129

130

131

132

133

134

135

136

137

138

139

140

141

142

143

144

145

146

147

148

149

150

151

152

153

154

155

156

157

7), 144.01 (phenyl C-1), 145.68 (phenyl C-3), 146.37 (quinoline C-2), 148.91(quinoline C-9), 152.06 (quinoline C-4), $166.33\left(\mathrm{C}=\mathrm{O}\right.$ ); HRESI-MS $m / z$ calcd for $[\mathrm{M}+\mathrm{H}]^{+} \mathrm{C}_{18} \mathrm{H}_{16} \mathrm{BrN}_{3} \mathrm{O}_{4} \mathrm{~S}$ : 450.0123, found: 450.0127 .

Ethyl 6-methoxy-4-((3-sulfamoylphenyl)amino)quinoline-3-carboxylate (5b)

Yellow solid, yield: $65 \%,{ }^{1} \mathrm{H}$ NMR (DMSO- $d 6,400 \mathrm{MHz}$ at $298 \mathrm{~K}$ ): 1.13 (t, $3 \mathrm{H}, J=6.8 \mathrm{~Hz}$, $\mathrm{CH}_{2} \mathrm{CH}_{3}$ ), 3.73 (s, $3 \mathrm{H}, \mathrm{OCH}_{3}$ ), 3.99 (q, $\left.2 \mathrm{H}, J=6.8 \mathrm{~Hz}, \mathrm{CH}_{2} \mathrm{CH}_{3}\right), 7.14-7.16(\mathrm{~m}, 1 \mathrm{H}$, phenyl H2), 7.33 (s, 2H, phenyl H-4), 7.42-7.48 (m, 5H, phenyl H-5,6, quinoline $\mathrm{H}-5,7,8), 7.91-7.93(\mathrm{~m}$, $1 \mathrm{H}$, quinoline $\mathrm{H}-2), 8.84(\mathrm{~s}, 1 \mathrm{H}, \mathrm{NH}) ;{ }^{13} \mathrm{C}$ NMR (DMSO-d6): $14.31\left(\mathrm{CH}_{2} \mathrm{CH}_{3}\right), 55.92\left(\mathrm{OCH}_{3}\right)$, $61.40\left(\mathrm{CH}_{2}\right), 103.51$ (quinoline C-5), 111.54 (phenyl C-2), 116.36 (phenyl C-4), 119.51 (phenyl C-6), 121.78 (quinoline C-3), 122.35 (quinoline C-10), 123.78 (quinoline C-7), 130.17 (phenyl C-5), 131.56 (quinoline C-8), 144.36 (quinoline C-9), 145.55 (phenyl C-3), 146.16 (phenyl C-1), 146.59 (quinoline $\mathrm{C}-2$ ), 148.90 (quinoline $\mathrm{C}-3$ ), 157.51 (quinoline $\mathrm{C}-6), 166.90(\mathrm{C}=\mathrm{O})$; HRESIMS $\underline{m / z}$ calcd for $[\mathrm{M}+\mathrm{H}]^{+} \mathrm{C}_{19} \mathrm{H}_{19} \mathrm{~N}_{3} \mathrm{O}_{5} \mathrm{~S}: 402.1124$, found: 402.1116 .

Ethyl 7-chloro-6-fluoro-4-((3-sulfamoylphenyl)amino)quinoline-3-carboxylate (5c)

Yellowish white solid, yield: $66 \%,{ }^{1} \mathrm{H}$ NMR (DMSO- $d 6,400 \mathrm{MHz}$ at $298 \mathrm{~K}$ ): 1.08 (t, 3H, $J=7.2$ $\left.\mathrm{Hz}, \mathrm{CH}_{2} \mathrm{CH}_{3}\right), 3.91\left(\mathrm{q}, 2 \mathrm{H}, J=7.2 \mathrm{~Hz}, \mathrm{CH}_{2} \mathrm{CH}_{3}\right), 7.18-7.20(\mathrm{~m}, 1 \mathrm{H}$, quinoline $\mathrm{H}-2), 7.45-7.49$ (m, 3H, phenyl H-4,5,6), $7.36(\mathrm{~s}, 2 \mathrm{H}), 8.18(\mathrm{~d}, 1 \mathrm{H}, J=11.2 \mathrm{~Hz}$, quinoline $\mathrm{H}-8), 8.25(\mathrm{~d}, 1 \mathrm{H} J=$ $7.6 \mathrm{~Hz}$, quinoline $\mathrm{H}-7), 8.91\left(\mathrm{~s}, 1 \mathrm{H}, \mathrm{SO}_{2} \mathrm{NH}\right), 9.68(\mathrm{~s}, 1 \mathrm{H}, \mathrm{NH}) ;{ }^{13} \mathrm{C}$ NMR (DMSO-d6): 14.22 $\left(\mathrm{CH}_{2} \mathrm{CH}_{3}\right), 61.58\left(\mathrm{CH}_{2}\right), 110.16,110.40$ (phenyl C-2), 111.47, 116.52 (phenyl C-4), 120.18, 121.24 (phenyl C-6), 121.85 (quinoline C-10), 124.41 (quinoline C-8), 130.29 (phenyl C-5), 131.58, 143.76 (phenyl C-3), 145.69 (phenyl C-1), 147.05 (quinoline C-2), 147.52 (quinoline C5), 152.27 (quinoline $\mathrm{C}-3$ ), 153.76 (quinoline $\mathrm{C}-7$ ), 156.21 (quinoline $\mathrm{C}-6$ ), $166.26(\mathrm{C}=\mathrm{O}$ ); HRESI-MS m/z calcd for $[\mathrm{M}+\mathrm{H}]+\mathrm{C}_{18} \mathrm{H}_{15} \mathrm{ClFN}_{3} \mathrm{O}_{4} \mathrm{~S}: 424.0534$, found: 424.0525 . Ethyl 6-Bromo-4-((3-(N-methylsulfamoyl)phenyl)amino)quinoline-3-carboxylate (5d) White solid, yield: $64 \%$, mp: $212.9-214.3{ }^{\circ} \mathrm{C},{ }^{1} \mathrm{H}$ NMR (DMSO- $\left.d 6,400 \mathrm{MHz}\right) \delta p p m: 1.08(\mathrm{t}$, $\left.3 \mathrm{H}, J=6.8 \mathrm{~Hz}, \mathrm{CH}_{2} \mathrm{CH}_{3}\right), 2.98\left(\mathrm{~s}, 3 \mathrm{H}, \mathrm{NHCH}_{3}\right) 3.91\left(\mathrm{q}, 2 \mathrm{H}, J=6.8 \mathrm{~Hz}, \mathrm{CH}_{2} \mathrm{CH}_{3}\right), 7.27$ (d, $1 \mathrm{H}$, $J=7.6 \mathrm{~Hz}$, benzenesulfonamide $\mathrm{H}-2), 7.95(\mathrm{~m}, 1 \mathrm{H}$, quinoline $\mathrm{H}-5), 8.40(\mathrm{~s}, 1 \mathrm{H}$, quinoline $\mathrm{H}-8)$, $8.48(\mathrm{~s}, 1 \mathrm{H}$, quinoline $\mathrm{H}-2), 8.89\left(\mathrm{~s}, 1 \mathrm{H}, \mathrm{SO}_{2} \mathrm{NH}\right), 9.64(\mathrm{~s}, 1 \mathrm{H}, \mathrm{NH}) ;{ }^{13} \mathrm{C}$ NMR (DMSO-d6, 100 MHz) $\delta$ ppm: $14.20\left(\mathrm{CH}_{3}\right), 29.05\left(\mathrm{~N}-\mathrm{CH}_{3}\right), 61.45\left(\mathrm{CH}_{2}\right), 111.61$ (benzenesulfonamide $\left.\mathrm{C}-2\right)$, 117.40 (benzenesulfonamide C-4), 119.76 (benzenesulfonamide C-6), 121.02 (quinoline C-3),

Peer) reviewing PDF | (2019:12:43714:2:1:NEW 26 Jan 2020) 
158122.53 (quinoline C-10), 123.04 (quinoline C-6), 126.69 (quinoline C-5), 130.56

159 (benzenesulfonamide C-5), 132.14 (quinoline C-8), 134.75 (quinoline C-7), 140.80

160 (benzenesulfonamide C-3), 144.35 (benzenesulfonamide C-1), 146.54 (quinoline C-2), 148.95

161 (quinoline C-9), 152.03 (quinoline $\mathrm{C}-4), 166.34(\mathrm{C}=\mathrm{O})$; HRESI-MS $m / z$ calcd for $[\mathrm{M}+\mathrm{H}]^{+}$

$162 \mathrm{C}_{19} \mathrm{H}_{19} \mathrm{BrN}_{3} \mathrm{O}_{4} \mathrm{~S}: 464.0280$, found: 464.0273 .

163

164

165

Ethyl 6-methoxy-4-((3-(methylsulfonamido)phenyl)amino)quinoline-3-carboxylate (5e)

166

Yellow solid, yield: 64\%, ${ }^{1} \mathrm{H}$ NMR (DMSO- $d 6,400 \mathrm{MHz}$ at $298 \mathrm{~K}$ ): 1.17 (t, $3 \mathrm{H}, J=7.2 \mathrm{~Hz}, \mathrm{CH}_{2}$ $\left.\mathrm{CH}_{3}\right), 2.96\left(\mathrm{~s}, 3 \mathrm{H}, \mathrm{SO}_{2} \mathrm{CH}_{3}\right), 3.71\left(\mathrm{~s}, 3 \mathrm{H}, \mathrm{OCH}_{3}\right), 3.99$ (q, $\left.2 \mathrm{H}, J=7.2 \mathrm{~Hz}, \mathrm{CH}_{2} \mathrm{CH}_{3}\right), 6.75-6.77$

167 (m, 1H, phenyl H-2), 6.88-6.90 (m, 2H, phenyl H-4,6), 7.22-7.26 (m, 1H, phenyl H-5), 7.40-7.45

168 (m, 2H, quinoline $\mathrm{H}-5,7), 7.89(\mathrm{~d}, J=8 \mathrm{~Hz}, 1 \mathrm{H}$, quinoline $\mathrm{H}-8), 8.80(\mathrm{~s}, 1 \mathrm{H}$, quinoline $\mathrm{H}-2)$, $9.52\left(\mathrm{~s}, 1 \mathrm{H}, \mathrm{SO}_{2} \mathrm{NH}\right), 9.74(\mathrm{~s}, 1 \mathrm{H}, \mathrm{NH}) ;{ }^{13} \mathrm{C} \mathrm{NMR}$ (DMSO-d6): $14.35\left(\mathrm{CH}_{2} \mathrm{CH}_{3}\right), 55.82\left(\mathrm{OCH}_{3}\right)$ $61.29\left(\mathrm{CH}_{2}\right), 103.88$ (quinoline $\left.\mathrm{C}-5\right), 110.41$ (phenyl C-2), 111.21 (phenyl C-4), 114.27 (phenyl C-6), 115.58 (quinoline C-3), 121.85 (quinoline C-10), 123.45 (quinoline C-7), 130.44 (phenyl C5), 139.84 (quinoline C-8), 144.51 (quinoline C-9), 146.11 (phenyl C-3), 147.58 (phenyl C-1), 148.88 (quinoline $\mathrm{C}-2$ ), 157.14 (quinoline $\mathrm{C}-3$ ), 150.45 (quinoline $\mathrm{C}-6$ ), 167.41( $\mathrm{C}=\mathrm{O}$ ); HRESI-

174 MS m/z calcd for $[\mathrm{M}+\mathrm{H}]^{+} \mathrm{C}_{20} \mathrm{H}_{21} \mathrm{~N}_{3} \mathrm{O}_{5} \mathrm{~S}: 416.1280$, found: 416.1278 .

175

176

Ethyl 7-chloro-6-fluoro-4-((3-(methylsulfonamido)phenyl)amino)quinoline-3-carboxylate (5f)

177

Yellow solid, yield: 55\%, ${ }^{1} \mathrm{H}$ NMR (DMSO- $d 6,400 \mathrm{MHz}$ at $298 \mathrm{~K}$ ): 1.12 (t, $3 \mathrm{H}, J=6.8 \mathrm{~Hz}, \mathrm{CH}_{2}$

178 $\left.\mathrm{CH}_{3}\right), 2.99$ (s, $\left.3 \mathrm{H}, \mathrm{SO}_{2} \mathrm{CH}_{3}\right), 3.92\left(\mathrm{q}, 2 \mathrm{H}, J=6.8 \mathrm{~Hz}, \mathrm{CH}_{2} \mathrm{CH}_{3}\right), 6.77(\mathrm{~d}, 1 \mathrm{H}, J=8 \mathrm{~Hz}$, phenyl H2), 6.90-6.93 (m, 2H, phenyl H-4,6), 7.23-7.27 (m, H, phenyl H-5), $8.12(\mathrm{~d}, 1 \mathrm{H}, J=11.2 \mathrm{~Hz}$, quinoline $\mathrm{H}-7), 8.21(\mathrm{~d}, J=6 \mathrm{~Hz}, 1 \mathrm{H}$, quinoline $\mathrm{H}-8), 8.87(\mathrm{~s}, 1 \mathrm{H}$, quinoline $\mathrm{H}-2), 9.63(\mathrm{~s}, 1 \mathrm{H}$, $\left.\mathrm{SO}_{2} \mathrm{NH}\right), 9.79$ (s, $\left.1 \mathrm{H}, \mathrm{NH}\right)$; HRESI-MS $\underline{m / z}$ calcd for $[\mathrm{M}+\mathrm{H}]^{+} \mathrm{C}_{19} \mathrm{H}_{17} \mathrm{ClFN}_{3} \mathrm{O}_{4} \mathrm{~S}: 438.0691$, found: 438.0687 .

183

Ethyl 6-Bromo-4-(3-methanesulfonylphenylamino)-quinoline-3-carboxylate (5g)

185

186

187

188

189

190

191

192

193

194

195

196
Yellow solid, yield: $67 \%$, mp: $183.4-184.5{ }^{\circ} \mathrm{C},{ }^{1} \mathrm{H}$ NMR (DMSO- $\left.d 6,400 \mathrm{MHz}\right) \delta p p m: 1.12$ (t, $\left.3 \mathrm{H}, J=7.2 \mathrm{~Hz}, \mathrm{CH}_{2} \mathrm{CH}_{3}\right), 2.98\left(\mathrm{~s}, 3 \mathrm{H}, \mathrm{SO}_{2} \mathrm{CH}_{3}\right) 3.93\left(\mathrm{q}, 2 \mathrm{H}, J=7.2 \mathrm{~Hz}, \mathrm{CH}_{2} \mathrm{CH}_{3}\right), 6.78(\mathrm{~s}, 1 \mathrm{H}$, phenyl H-2), 6.91-6.94 (m, 2H, phenyl H-4,6), $7.24(\mathrm{~m}, 1 \mathrm{H}$, phenyl H-5), $7.90(\mathrm{~m}, 2 \mathrm{H}$, quinoline $\mathrm{H}-5,7), 8.40(\mathrm{~m}, 1 \mathrm{H}$, quinoline $\mathrm{H}-8), 8.88(\mathrm{~s}, 1 \mathrm{H}$, quinoline $\mathrm{H}-2), 9.67(\mathrm{~s}, 1 \mathrm{H}, \mathrm{NH}) ;{ }^{13} \mathrm{C} \mathrm{NMR}$ (DMSO-d6, $100 \mathrm{MHz})$ sppm: $14.29\left(\mathrm{CH}_{3}\right), 39.35\left(\mathrm{SO}_{2} \mathrm{CH}_{3}\right), 61.41\left(\mathrm{CH}_{2}\right), 110.54$, (phenyl C-2), 111.07 (phenyl C-4), 114.67 (phenyl C-6), 115.17 (quinoline C-7), 119.24 (quinoline C-3), 122.54 (quinoline C-10), 127.01 (quinoline C-6), 130.54 (quinoline C-5), 132.07 (phenyl C-5), 134.59 (quinoline C-8), 140.10 (phenyl C-3), 144.10 (phenyl C-1), 147.47 (quinoline C-2), 148.96 (quinoline $\mathrm{C}-9), 151.91$ (quinoline $\mathrm{C}-4), 166.90(\mathrm{C}=\mathrm{O})$; HRESI-MS $\mathrm{m} / \mathrm{z}$ calcd for $[\mathrm{M}+\mathrm{H}]^{+} \mathrm{C}_{19} \mathrm{H}_{19} \mathrm{BrN}_{3} \mathrm{O}_{4} \mathrm{~S}: 464.0280$, found: 464.0276 .

Ethyl 6-methoxy-4-((3-(methylsulfonyl)phenyl)amino)quinoline-3-carboxylate (5h) 
197 Yellow solid, yield: $62 \%,{ }^{1} \mathrm{H}$ NMR (DMSO- $d 6,400 \mathrm{MHz}$ at $298 \mathrm{~K}$ ): $1.13(\mathrm{t}, 3 \mathrm{H}, J=7.2 \mathrm{~Hz}$,

198

199

200

201

202

203

204

205

206

207

208

209

210

211

212

213

214

215

216

217

218

219

220

221

222

223

224

225

226

227

228

229

230

231

232

233

234

235

236

$\left.\mathrm{CH}_{2} \mathrm{CH}_{3}\right), 2.39$ (s, $3 \mathrm{H}, \mathrm{NHCH}_{3}$ ), $3.75\left(\mathrm{~s}, 3 \mathrm{H}, \mathrm{OCH}_{3}\right), 3.99$ (q, $\left.2 \mathrm{H}, J=7.2 \mathrm{~Hz}, \mathrm{CH}_{2} \mathrm{CH}_{3}\right), 7.24$ (d, $1 \mathrm{H}, J=7.6 \mathrm{~Hz}$, phenyl H-2), 7.36-7.52 (m, 6H, phenyl H-4,5,6, quinoline $\mathrm{H}-5,7,8), 7.95(\mathrm{~s}, 1 \mathrm{H}$, quinoline $\mathrm{H}-2), 8.85\left(\mathrm{~s}, 1 \mathrm{H}, \mathrm{SO}_{2} \mathrm{NH}\right), 9.57(\mathrm{~s}, 1 \mathrm{H}, \mathrm{NH}) ;{ }^{13} \mathrm{C} \mathrm{NMR}$ (DMSO-d6): $14.28\left(\mathrm{CH}_{2}\right.$ $\left.\mathrm{CH}_{3}\right), 28.99\left(\mathrm{NHCH}_{3}\right), 55.91\left(\mathrm{OCH}_{3}\right) 61.35\left(\mathrm{CH}_{2}\right), 103.47$ (quinoline C-5), 111.69 (phenyl C-2), 117.23 (phenyl C-4), 120.47 (phenyl C-6), 122.39 (quinoline C-3), 122.52 (quinoline C-10), 123.73 (quinoline C-7), 130.49 (phenyl C-5), 131.60 (quinoline C-8), 140.72 (quinoline C-9), 144.77 (phenyl C-3), 146.17 (phenyl C-1), 146.50 (quinoline C-2), 148.92 (quinoline C-3), 157.54 (quinoline C-6), $166.86(\mathrm{C}=\mathrm{O})$; HRESI-MS m/z calcd for $[\mathrm{M}+\mathrm{H}]^{+} \mathrm{C}_{20} \mathrm{H}_{21} \mathrm{~N}_{3} \mathrm{O}_{5} \mathrm{~S}$ : 416.1280, found: 416.1277 .

Ethyl 7-chloro-6-fluoro-4-((3-(methylsulfonyl)phenyl)amino)quinoline-3-carboxylate (5i) Yellow solid, yield: $58 \%,{ }^{1} \mathrm{H}$ NMR (DMSO- $d 6,400 \mathrm{MHz}$ at $298 \mathrm{~K}$ ): 1.07 (t, $3 \mathrm{H} J=6.8 \mathrm{~Hz}$, $\left.\mathrm{CH}_{2} \mathrm{CH}_{3}\right), 2.40$ (s, $\left.3 \mathrm{H}, \mathrm{NHCH}_{3}\right), 3.90$ (q, $\left.2 \mathrm{H}, J=6.8 \mathrm{~Hz}, \mathrm{CH}_{2} \mathrm{CH}_{3}\right), 7.27$ (d, $1 \mathrm{H}, J=7.6 \mathrm{~Hz}$ phenyl H-2), 7.41(s, 1H, phenyl H-5), 7.49-7.53 (m, 2H, phenyl H-4,6), $8.19(\mathrm{~d}, 1 \mathrm{H}, J=11.6 \mathrm{~Hz}$ quinoline $\mathrm{H}-7), 8.26(\mathrm{~d}, 1 \mathrm{H}, J=7.2 \mathrm{~Hz}$ quinoline $\mathrm{H}-8), 8.91(\mathrm{~s}, 1 \mathrm{H}$, quinoline $\mathrm{H}-2), 9.70(\mathrm{~s}, 1 \mathrm{H}$, $\left.\mathrm{SO}_{2} \mathrm{NH}\right), 9.79(\mathrm{~s}, 1 \mathrm{H}, \mathrm{NH}) ;{ }^{13} \mathrm{C} \mathrm{NMR}$ (DMSO-d6): $14.19\left(\mathrm{CH}_{2} \mathrm{CH}_{3}\right), 29.00\left(\mathrm{NHCH}_{3}\right), 61.52$ $\left(\mathrm{CH}_{2}\right), 110.18,110.42$ (phenyl C-2), 111.56,117.44 (phenyl C-4), 121.17, 121.25 (phenyl C-6), 121.33 (quinoline C-10), 122.63 (quinoline C-8), 125.22, 125.43,130.61 (phenyl C-5), 131.58, 140.89, 144.09 (phenyl C-3), 146.97 (phenyl C-1), 147.02 (quinoline C-2), 147.54 (quinoline C5), 152.26 (quinoline $\mathrm{C}-3$ ), 153.76 (quinoline $\mathrm{C}-7$ ), 156.21 (quinoline $\mathrm{C}-6$ ), $166.24(\mathrm{C}=\mathrm{O}$ ). HRESI-MS $\underline{m / z}$ calcd for $[\mathrm{M}+\mathrm{H}]^{+} \mathrm{C}_{19} \mathrm{H}_{17} \mathrm{Cl} \mathrm{FN}_{3} \mathrm{O}_{4} \mathrm{~S}$ : 438.0691 , found: 438.0693 .

\section{In vitro kinase inhibition assay}

Reaction Biology Corp. Kinase HotSpotSM service (http://www.reactionbiology.com) was used for the screening of tested compounds. Kinase Profiling is 10 dose $\mathrm{IC}_{50}$ singlet assay. Activity of kinases were assessed by the HotSpot assay platform, which contained specific kinase/substrate pairs along with required cofactors (Abdelazem et al. 2015; Abdelazem et al. 2016; Al-Sanea et al. 2016a; Al-Sanea et al. 2015; Al-Sanea et al. 2013; Al-Sanea et al. 2015; Park et al. 2014).

\section{Docking studies}

Molecular Operating Environment (MOE version 2008.10) by Chemical Computing Group (CCG) was used for the docking studies (Inc. 2016). The protein preparation steps involved 3D protonation, energy minimization, and active site identification. The X-ray crystallographic structure of CDK8/CycC enzyme co-crystallized with Senexin A (PDB code $4 \mathrm{f} 7 \mathrm{~s}$ ) was obtained from the Protein Data Bank (Schneider et al. 2013). The enzyme was prepared for virtual docking studies where: (i) the ligand molecule with any existing solvent molecules were removed. (ii) Hydrogen atoms were added to the structure with their standard geometry. To visualize the binding pocket, alpha spheres were created followed by the generation of dummy atoms on the centers of these spheres. The pocket was found to be a deep cavity lined with the

PeerJ reviewing PDF | (2019:12:43714:2:1:NEW 26 Jan 2020) 
237 amino acid residues including both hydrophobic and hydrophilic amino acids. Energy

238 minimization tool MOPAC 7.0 was applied for the tilted compounds.. (iii) MOE Alpha Site

239 Finder was used for the active sites search and dummy atoms were created from the obtained

240 alpha spheres. The obtained ligand-enzyme complex model was then used in calculating the

241 energy parameters using MMFF94x force field energy calculation and predicting the ligand-

242 enzyme interactions.

243

244

\section{Results}

245

246

247

248

249

The methods followed for the synthesis of the target compounds 5a-i are represented in Scheme

250

1. Anilines 1a-c were firstly refluxed in ethanol with diethyl ethoxymethylenemalonate to provide substituted phenylaminomethylenemalonates $\mathbf{2 a - c}$. Compounds $\mathbf{2 a - c}$ were cyclized

251 thermally in diphenyl ether to the corresponding 4-oxo-1,4-dihydroquinolines 3a-c. Under the

252 anhydrous condition, quinolines $3 \mathbf{a}-\mathbf{c}$ were chlorinated via heating with excess of $\mathrm{POCl}_{3}$ to provide the key intermediates $\mathbf{4 a - c}$, as reported previously (Al-Sanea et al. 2019; Medapi et al. 2015; Rivilli et al. 2018). The target compounds 5a-i were achieved through microwave-assisted nucleophilic substitution reaction of 3-amino-N-methylbenzenesulfonamide, 3-

256

257 aminobenzenesulfonamide and $\mathrm{N}-(3$-phenyl)methanesulfonamide with the appropriate key intermediate $\mathbf{4 a - c}$ in ethanol (Al-Sanea et al. 2019).

258

259

260

261

262

263

264

265

266

267

268

269

270

\section{CDK8/CycC complex inhibition}

The newly prepared phenylaminoquinolines $\mathbf{5 a}-\mathbf{i}$ were biologically evaluated as potential $\mathrm{CDK} 8 / \mathrm{CycC}$ complex inhibitors. The percentage enzyme inhibition and half-maximal inhibitory concentration data of the target compounds with phenylaminoquinoline core structure and Staurosporine (as a standard inhibitor) against $\mathrm{CDK} 8 / \mathrm{CycC}$ are summarized in Table 1.

\section{Molecular docking}

For designing $\mathrm{CDK} 8 / \mathrm{CycC}$ type I inhibitor, targeting the hinge residue is essential to inhibit the kinase activity of the complex. To visualize the binding interactions between the promising biologically active compound $\mathbf{5 d}$, we obtained a co-crystal structure of 6-isocyano-Nphenethylquinazolin-4-amine (Senexin A ) in complexation with CDK8:Cyclin C with the DMG

271 motif in the "in" conformation at $2.2 \AA$ resolution (PDB : 4F7S).

272

\section{Discussion}

274 Chemistry

275 
276 Based on ${ }^{1} \mathrm{H}-\mathrm{NMR},{ }^{13} \mathrm{C}-\mathrm{NMR}$ spectroscopic data and high-resolution mass spectroscopy

277 (HRMS), the structures of all newly synthesized compounds were confirmed. ${ }^{1} \mathrm{HNMR}$ spectra of

278 all finals 5a-i showed new characteristic signals at $\delta 7.33-7.37 \mathrm{ppm}$, and $9.68-10.25 \mathrm{ppm}$

279 corresponding to $\mathrm{NH}_{2}$ and $\mathrm{NH}$ groups, respectively, that distinguished the finals $\mathbf{5 a - i}$ from

280 chloroquinolines 4a-c. For compound 5d, three characteristic signals at $\delta 2.42,8.94$, and 9.64

281 ppm were displayed and assigned to $-\mathrm{NHCH}_{3},-\mathrm{SO}_{2} \mathrm{NH}$ - and - $\mathrm{NH}$ - protons, respectively.

282

283

284

285

286

287

288

289

290

291

292

293

294

295

296

297

298

299

300

301

302

303

304

305

306

307

308

309

310

311

\section{CDK8/CycC complex inhibition}

According to the inhibition data stated in Table 1, the following structure-activity relationship (SAR) notes are described as follows.

The methanesulfonamide analogue $\mathbf{5 d}$ showed maximal potency among all final compounds with submicromolar activity and $\mathrm{IC}_{50}$ value of $0.639 \mu \mathrm{M}$, whereas, the corresponding primary benzenesulfonamide analogue 5a exhibited a 6-fold decrease in potency $\left(\mathrm{IC}_{50}=3.98 \mu \mathrm{M}\right)$. On the contrary, the corresponding substituted benzenesulfonamide analogue $(\mathbf{5 g})$ exhibited no CDK8/CycC complex inhibitory activity, confirming the crucial role of PKa values of sulfonamide groups for the intrinsic activity of pharmacophore of the phenylaminoquinoline scaffold-based compounds. Moreover, the primary benzenesulfonamide analogues (5a \& $\mathbf{5 b}$ ) exhibited single digit micromolar potency in inhibiting the $\mathrm{CDK} 8 / \mathrm{CycC}$. Whereas, methanesulphonamide analogues ( $\mathbf{5} \mathbf{d} \& \mathbf{5 e})$ showed superior potency with $\mathrm{IC}_{50}$ values of 0.639 and $1.42 \mu \mathrm{M}$, respectively. Noteworthy, all 7-chloro-6-fluoro substituted quinolines $(\mathbf{5 c}, \mathbf{5 f}, \mathbf{5 i})$ failed to inhibit the $\mathrm{CDK} 8 / \mathrm{CycC}$ enzyme, signifying the remarkable adverse effects of some quinoline substituents on the binding interaction, and hence the intrinsic activity. Therefore, concerning the influence of substitution of the quinoline moiety, the inhibitory activities increased in the order of 7-Cl-6-F $<6-\mathrm{OCH}_{3}<6-\mathrm{Br}$.

\section{Molecular docking}

The virtual docking study showed how the compound $\mathbf{5 d}$ in the 3D docking pose can anchor in the kinase deep pocket and extended with diverse functional groups toward the hinge region and the front pocket. Two direct hydrogen bonds are formed between the inhibitor $\mathbf{5 d}$ and the kinase domain of $\mathrm{CDK} 8 / \mathrm{CycC}$. The quinoline $\mathrm{N}$ forms an essential single $\mathrm{H}$-bond with the backbone nitrogen of Ala100CDK8 on the hinge region. The sulfonyl $\mathrm{O}$ forms the second direct $\mathrm{H}$-bond to the backbone $\mathrm{N}$ of Asp173CDK8 of the DMG motif. Moreover, $\pi-\pi$ stacking interaction with the gatekeeper residue (Phe97CDK8) and VDW interactions with several residues at the ATP binding pocket (Ala172CDK8, Ala50CDK8, Val27CDK8, Leu158CDK8, Val35CDK8,

312

313

314 Conclusions Tyr99CDK8, Ile79CDK8) were shown as depicted in Figure 1.

313 
315 We have demonstrated a new series of phenylaminoquinoline core structure-based compounds

316 5a-i have been synthesized and biologically evaluated as potential CDK8/CycC inhibitors. The

317 methanesulfonamide analogues ( $\mathbf{5 d} \& \mathbf{5 e}$ ) proved to be the most potent compounds in

318 suppressing $\mathrm{CDK} 8 / \mathrm{CycC}$ enzyme, whereas, the un/substitutes benzenesulfonamide analogues

319 showed inferior (or no) activity, demonstrating the advantage of highly acidic $\mathrm{NH}$ of

320 sulfonamide moiety. Careful selection of quinoline moiety substituents is highly recommended,

321 as 7-chloro-6-fluoro bearing analogues $(\mathbf{5 c} \& \mathbf{5 f})$ showed no inhibition. Moreover, the secondary

322 benzenesulfonamide analogues should be avoided, as they showed no inhibitory activities. We

323 have discovered the most potent analogue $\mathbf{5 d}$ with submicromolar potency against $\mathrm{CDK} 8 / \mathrm{CycC}$

$324\left(\mathrm{IC}_{50}=0.639 \mu \mathrm{M}\right)$ and it can be prepared in four steps with an overall yield of $64 \%$ making it

325 suitable for further investigations. Larger library of phenylaminoquinoline scaffold-based

326 analogues are going to be prepared by our team to establish detailed and distinguished SARs.

327 In summary, the novel ((3-(N-methylsulfamoyl)phenyl)amino)quinoline derivatives exhibited

328 potent $\mathrm{CDK} 8 / \mathrm{CycC}$ inhibitory activity. Developing inhibitors targeting $\mathrm{CDK} 8 / \mathrm{CycC}$ offers an

329 exciting approach for treatment of human carcinomas.

330

331

\section{Acknowledgments}

332

333

I would like to express my special appreciation and thanks to my colleague Dr. Ahmed

Elkamhawy, you have been a great research collaborator for me in this project.

334

335

336

337

338

339

340

341

342

343

344

345

346

347

\section{References}

Abdelazem AZ, Al-Sanea MM, Park BS, Park HM, Yoo KH, Sim T, Park JB, Lee S-H, and Lee $\mathrm{SH}$. 2015. Synthesis and biological evaluation of new pyrazol-4-ylpyrimidine derivatives as potential ROS1 kinase inhibitors. European journal of medicinal chemistry 90:195208.

Abdelazem AZ, Al-Sanea MM, Park H-M, and Lee SH. 2016. Synthesis of new diarylamides with pyrimidinyl pyridine scaffold and evaluation of their anti-proliferative effect on cancer cell lines. Bioorganic \& medicinal chemistry letters 26:1301-1304.

Adler AS, McCleland ML, Truong T, Lau S, Modrusan Z, Soukup TM, Roose-Girma M, Blackwood EM, and Firestein R. 2012. CDK8 maintains tumor dedifferentiation and embryonic stem cell pluripotency. Cancer research 72:2129-2139.

Al-Sanea M, Abdelazem A, Park B, Yoo K, Sim T, Kwon Y, and Lee S. 2016a. ROS1 kinase inhibitors for molecular-targeted therapies. Current medicinal chemistry 23:142-160.

Al-Sanea M, Elkamhawy A, Zakaria A, Park B, Kwon Y, Lee S, Lee S, and Kim I. 2015. Synthesis and in vitro screening of phenylbipyridinylpyrazole derivatives as potential antiproliferative agents. Molecules 20:1031-1045.

Al-Sanea MM, Abdelazem AZ, Park BS, Yoo KH, Sim T, Kwon YJ, and Lee SH. 2016b. ROS1 Kinase Inhibitors for Molecular-Targeted Therapies. Curr Med Chem 23:142-160.

Al-Sanea MM, El-Deeb IM, and Lee SH. 2013. Design, synthesis and in vitro screening of new $1 \mathrm{H}$-pyrazole and 1, 2-isoxazole derivatives as potential inhibitors for ROS and MAPK14 kinases. Bull Korean Chem Soc 34:437.

Al-Sanea MM, Elkamhawy A, Paik S, Bua S, Ha Lee S, Abdelgawad MA, Roh EJ, Eldehna WM, and Supuran CT. 2019. Synthesis and biological evaluation of novel 3-(quinolin-4ylamino) benzenesulfonamidesAQ3 as carbonic anhydrase isoforms I and II inhibitors. Journal of enzyme inhibition and medicinal chemistry 34:1457-1464.

Peer) reviewing PDF | (2019:12:43714:2:1:NEW 26 Jan 2020) 
360

361

362

363

364

365

366

367

368

369

370

371

372

373

374

375

376

377

378

379

380

381

382

383

384

385

386

387

388

389

390

391

392

393

394

395

396

397

398

399

400

401

402

403

404

405

406

407

408

409

Al-Sanea MM, Park BS, Abdelazem AZ, Selim KB, Yoo KH, Sim T, Tae JS, and Lee SH. 2015. Optimization of Bipyridinyl Pyrazole Scaffolds via Design, Synthesis and Screening of a New Series of ROS1 Kinase-modulating Compounds. Bulletin of the Korean Chemical Society 36:305-311.

Cee VJ, Chen DYK, Lee MR, and Nicolaou KeC. 2009. Cortistatin A is a High-Affinity Ligand of Protein Kinases ROCK, CDK8, and CDK11. Angewandte Chemie International Edition 48:8952-8957.

Chen W, Ren X, and Chang CeA. 2019. Discovery of CDK8/CycC Ligands with a New Virtual Screening Tool. ChemMedChem 14:107-118.

Crown J. 2017. CDK8: a new breast cancer target. Oncotarget 8:14269.

Firestein R, Bass AJ, Kim SY, Dunn IF, Silver SJ, Guney I, Freed E, Ligon AH, Vena N, and Ogino S. 2008. CDK8 is a colorectal cancer oncogene that regulates $\beta$-catenin activity. Nature 455:547.

He L-J, Zhu Y-B, Fan Q-Z, Miao D-D, Zhang S-P, Liu X-P, and Zhang C. 2019. Shape-based virtual screen for the discovery of novel CDK8 inhibitor chemotypes. Bioorganic \& medicinal chemistry letters 29:549-555.

Inc. CCG. 2016. Molecular operating environment (MOE). Chemical Computing Group Inc 1010 Sherbooke St. West, Suite\# 910, Montreal ....

Kapoor A, Goldberg MS, Cumberland LK, Ratnakumar K, Segura MF, Emanuel PO, Menendez S, Vardabasso C, LeRoy G, and Vidal Cl. 2010. The histone variant macroH2A suppresses melanoma progression through regulation of CDK8. Nature 468:1105.

Kim S, Xu X, Hecht A, and Boyer TG. 2006. Mediator is a transducer of Wnt/ $\beta$-catenin signaling. Journal of Biological Chemistry 281:14066-14075.

Knuesel MT, Meyer KD, Bernecky C, and Taatjes DJ. 2009a. The human CDK8 subcomplex is a molecular switch that controls Mediator coactivator function. Genes \& development 23:439-451.

Knuesel MT, Meyer KD, Donner AJ, Espinosa JM, and Taatjes DJ. 2009b. The human CDK8 subcomplex is a histone kinase that requires Med12 for activity and can function independently of mediator. Molecular and cellular biology 29:650-661.

Li J, Li X, Kong X, Luo Q, Zhang J, and Fang L. 2014a. MiRNA-26b inhibits cellular proliferation by targeting CDK8 in breast cancer. International journal of clinical and experimental medicine 7:558.

Li N, Fassl A, Chick J, Inuzuka H, Li X, Mansour MR, Liu L, Wang H, King B, and Shaik S. 2014b. Cyclin C is a haploinsufficient tumour suppressor. Nature cell biology 16:1080.

Malik S, and Roeder RG. 2005. Dynamic regulation of pol II transcription by the mammalian Mediator complex. Trends in biochemical sciences 30:256-263.

McDermott MS, Chumanevich AA, Lim C-u, Liang J, Chen M, Altilia S, Oliver D, Rae JM, Shtutman M, and Kiaris H. 2017. Inhibition of CDK8 mediator kinase suppresses estrogen dependent transcription and the growth of estrogen receptor positive breast cancer. Oncotarget 8:12558.

Medapi B, Suryadevara P, Renuka J, Sridevi JP, Yogeeswari P, and Sriram D. 2015. 4Aminoquinoline derivatives as novel Mycobacterium tuberculosis GyrB inhibitors: structural optimization, synthesis and biological evaluation. European journal of medicinal chemistry 103:1-16.

Nemet J, Jelicic B, Rubelj I, and Sopta M. 2014. The two faces of Cdk8, a positive/negative regulator of transcription. Biochimie 97:22-27.

Obaya A, and Sedivy JM. 2002. Regulation of cyclin-Cdk activity in mammalian cells. Cellular and Molecular Life Sciences CMLS 59:126-142.

Park BS, Al-Sanea MM, Abdelazem AZ, Park HM, Roh EJ, Park H-M, Yoo KH, Sim T, Tae JS, and Lee SH. 2014. Structure-based optimization and biological evaluation of

Peer) reviewing PDF | (2019:12:43714:2:1:NEW 26 Jan 2020) 
410

411

412

413

414

415

416

417

418

419

420

421

422

423

424

425

426

427

428

429

430

431

432

433

434

435

436

437

438

439

440

441

442

443

444

445

446

trisubstituted pyrazole as a core structure of potent ROS1 kinase inhibitors. Bioorganic \& medicinal chemistry 22:3871-3878.

Pelish HE, Liau BB, Nitulescu II, Tangpeerachaikul A, Poss ZC, Da Silva DH, Caruso BT, Arefolov A, Fadeyi O, and Christie AL. 2015. Mediator kinase inhibition further activates super-enhancer-associated genes in AML. Nature 526:273.

Rivilli MJL, Turina AV, Bignante EA, Molina VH, Perillo MA, Briñon MC, and Moyano EL. 2018. Synthesis and pharmacological evaluation of pyrazolo [4, 3-c] quinolinones as high affinity GABAA-R ligands and potential anxiolytics. Bioorganic \& medicinal chemistry 26:3967-3974.

Roninson IB, Győrffy B, Mack ZT, Shtil AA, Shtutman MS, Chen M, and Broude EV. 2019. Identifying cancers impacted by CDK8/19. Cells 8:821.

Rzymski T, Mikula M, Wiklik K, and Brzózka K. 2015. CDK8 kinase-An emerging target in targeted cancer therapy. Biochimica et Biophysica Acta (BBA)-Proteins and Proteomics 1854:1617-1629.

Rzymski T, Mikula M, Żyłkiewicz E, Dreas A, Wiklik K, Gołas A, Wójcik K, Masiejczyk M, Wróbel A, and Dolata I. 2017. SEL120-34A is a novel CDK8 inhibitor active in AML cells with high levels of serine phosphorylation of STAT1 and STAT5 transactivation domains. Oncotarget 8:33779.

Sánchez-Martínez C, Lallena MJ, Sanfeliciano SG, and de Dios A. 2019. Cyclin dependent kinase (CDK) inhibitors as anticancer drugs: Recent advances (2015-2019). Bioorganic \& medicinal chemistry letters: 126637.

Satyanarayana A, and Kaldis P. 2009. Mammalian cell-cycle regulation: several Cdks, numerous cyclins and diverse compensatory mechanisms. Oncogene 28:2925.

Schiemann K, Mallinger A, Wienke D, Esdar C, Poeschke O, Busch M, Rohdich F, Eccles SA, Schneider R, and Raynaud FI. 2016. Discovery of potent and selective CDK8 inhibitors from an HSP90 pharmacophore. Bioorganic \& medicinal chemistry letters 26:1443-1451.

Schneider E, Böttcher J, Blaesse M, Neumann L, Huber R, and Maskos K. 2011. The structure of CDK8/CycC implicates specificity in the CDK/cyclin family and reveals interaction with a deep pocket binder. Journal of molecular biology 412:251-266.

Schneider EV, Böttcher J, Huber R, Maskos K, and Neumann L. 2013. Structure-kinetic relationship study of $\mathrm{CDK} 8 / \mathrm{CycC}$ specific compounds. Proceedings of the National Academy of Sciences 110:8081-8086.

Sears RC, and Nevins JR. 2002. Signaling networks that link cell proliferation and cell fate. Journal of Biological Chemistry 277:11617-11620.

$\mathrm{Xu} \mathrm{W}$, and Ji J-Y. 2011. Dysregulation of CDK8 and Cyclin C in tumorigenesis. Journal of Genetics and Genomics 38:439-452. 


\section{Figure 1}

A: Predicted 2D presentation of ligand binding modes of compound $5 d$ in the kinase domain of CDK8/CycC active pocket. B: Predicted 3D presentation of ligand binding pose of $5 d$ in the active site of $\mathrm{CDK} 8 / \mathrm{CycC}$ from the crystal structure 4F7S.

Discovery Studio Visualizer prepared 3D presentation by which the interacting residues are shown in lines; dotted lines are used to visualize the protein-ligand interactions.

A

Met

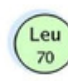

A Ala

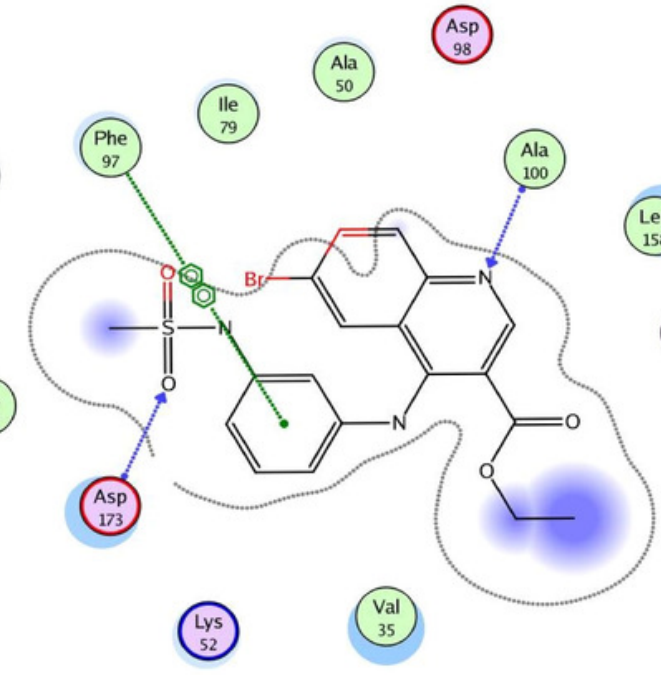

(Arg)
B

Tyr
99
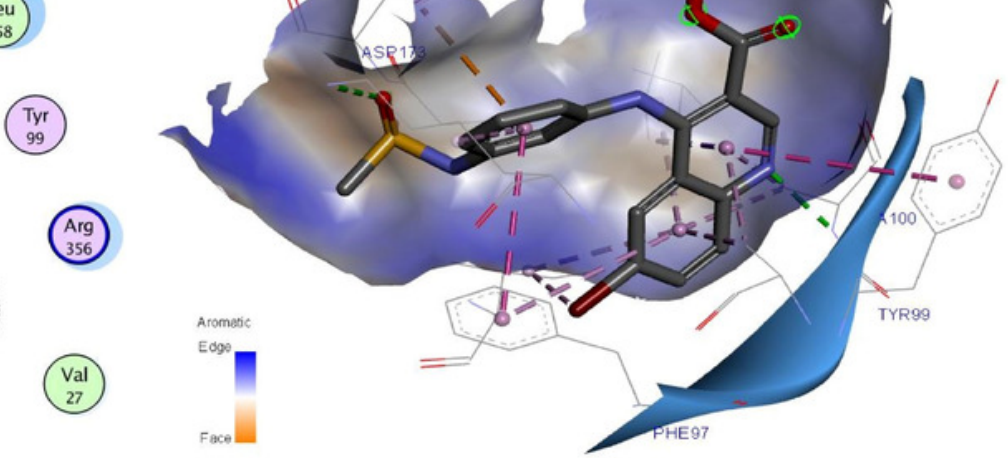
Figure 2

Synthesis of target quinolines $5 a-i$

Reagents and conditions: (i) Diethyl ethoxymethylenemalonate / Ethanol / reflux 1hr; (ii) Diphenyl ether / $250{ }^{\circ} \mathrm{C} / 45 \mathrm{~min}$; (iii) $\mathrm{POCl}_{3} /$ reflux $1 \mathrm{hr}$; (iv) Absolute ethanol/ reflux $4 \mathrm{hr}$.<smiles>[R][R]1ccc(N)c(Nc2c(C(=O)OCC)cnc3ccc([R])cc23)c1Nc1cccc([R])c1</smiles> 


\section{Table $\mathbf{1}$ (on next page)}

Inhibition data of CDK8/CycC for compounds 5a-i using Staurosporine as a standard inhibitor. 
2 Table 1. Inhibition data of $\mathrm{CDK} 8 / \mathrm{CycC}$ for compounds 5a-i, using Staurosporine as a standard 3 inhibitor.

4

\begin{tabular}{|c|c|c|c|c|}
\hline Comp. & $\mathbf{R}$ & $\mathbf{R}^{\prime}$ & $\begin{array}{c}\text { \% enzyme inhibition } \pm \mathbf{S D} \\
\mathbf{( 2 0} \boldsymbol{\mu M} \mathbf{)}\end{array}$ & $\mathbf{I C}_{\mathbf{5 0}}(\boldsymbol{\mu M M})$ \\
\hline $\mathbf{5 a}$ & $6-\mathrm{Br}$ & $\mathrm{SO}_{2} \mathrm{NH}_{2}$ & $83.91 \pm 1.83$ & 3.98 \\
\hline $\mathbf{5 b}$ & $6-\mathrm{OCH}$ & $\mathrm{SO}_{2} \mathrm{NH}_{2}$ & $63.365 \pm 2.31$ & 5.34 \\
\hline $\mathbf{5 c}$ & $7-\mathrm{Cl}-6-\mathrm{F}$ & $\mathrm{SO}_{2} \mathrm{NH}_{2}$ & $37.925 \pm 1.31$ & No inhibition \\
\hline $\mathbf{5 d}$ & $6-\mathrm{Br}$ & $\mathrm{NHSO}_{2} \mathrm{CH}_{3}$ & $88.59 \pm 0.97$ & 0.639 \\
\hline $\mathbf{5 e}$ & $6-\mathrm{OCH}$ & $\mathrm{NHSO}_{2} \mathrm{CH}_{3}$ & $85.875 \pm 0.94$ & 1.42 \\
\hline $\mathbf{5 f}$ & $7-\mathrm{Cl}-6-\mathrm{F}$ & $\mathrm{NHSO}_{2} \mathrm{CH}_{3}$ & $27.225 \pm 8.46$ & No inhibition \\
\hline $\mathbf{5 g}$ & $6-\mathrm{Br}$ & $\mathrm{SO}_{2} \mathrm{NHCH}_{3}$ & $22.425 \pm 1.02$ & No inhibition \\
\hline $\mathbf{5 h}$ & $6-\mathrm{OCH}$ & $\mathrm{SO}_{2} \mathrm{NHCH}_{3}$ & $18.655 \pm 0.48$ & No inhibition \\
\hline $\mathbf{5 i}$ & $7-\mathrm{Cl}-6-\mathrm{F}$ & $\mathrm{SO}_{2} \mathrm{NHCH}_{3}$ & $31.96 \pm 0.82$ & No inhibition \\
\hline \multicolumn{5}{|c}{$\mathrm{Staurosporine}$} \\
\hline
\end{tabular}

